\title{
CONVEXITY AT INFINITY AND BOUNDED HARMONIC FUNCTIONS
}

\author{
Albert Borbély
}

\begin{abstract}
It is shown that a complete simply connected negatively curved manifold supports nontrivial bounded harmonic functions if the singular set of the ideal boundary is disconnected.
\end{abstract}

\section{INTRODUCTION}

Harmonic functions on complete simply connected manifolds with negative curvature bounded away from 0 have been studied for some time. If the manifold has pinched negative curvature, that is, all the sectional curvatures lie between two negative constants, several basic questions have been solved $[2,3,4,5,7,10,11]$. However, in the general situation the existence of nontrivial bounded harmonic functions is still an open problem.

Throughout the paper let $M$ be a complete simply connected manifold with sectional curvatures $k \leqslant-1$. There is a natural compactification $\bar{M}=M \cup S_{\infty}(M)$ of such a manifold, where $S_{\infty}(M)$ denotes the ideal boundary and the topology is the usual cone topology (for details see [8]). In this situation the only existence theorem for nontrivial bounded harmonic functions is due to Choi [7] and it may be formulated as follows.

THEOREM A. Let $M$ be a complete simply connected manifold with sectional curvatures $k \leqslant-1$. Assume that $M$ can be written as a nontrivial union of two convex sets, that is, there are proper convex subsets $F_{a}$ and $F_{b}$ of $M$ such that $M=F_{a} \cup F_{b}$. Then there is a nontrivial bounded harmonic function on $M$.

While the condition of Theorem $\mathrm{A}$ is always satisfied on a manifold $M$ with pinched negative curvature [3] (or if the curvature has an exponential growth [5]), in general this is not the case. It was shown recently, that there are complete simply connected manifolds with sectional curvature $k<-1$ and a point $P$ on the ideal boundary with the property that the convex hull of every neighbourhood of $P$ is the whole manifold (see $[1,6]$ ). Moreover, there are manifolds such that every point on the ideal boundary has this property [1]. Clearly, Theorem A cannot be applied to the latter.

Received 12th August, 1996

Copyright Clearance Centre, Inc. Serial-fee code: 0004-9729/97 \$A2.00+0.00. 
We call a point $P \in S_{\infty}(M)$ on the ideal boundary singular if it has the above property, that is, the convex hull of every neighbourhood of $P$ is the whole manifold. The singular set, $S(M)$, of $M$ is the collection of singular ideal points. Clearly, the singular set must be closed in the cone topology. The aim of this note is to prove the following theorem about the singular set and harmonic functions.

THEOREM 1. Let $M$ be a complete simply connected manifold with sectional curvatures $k \leqslant-1$. Assume that the singular set $S(M)$ of $M$ is disconnected. Then

(a) the manifold supports nontrivial bounded harmonic functions.

(b) the interior (in $S_{\infty}(M)$ ) of $S(M)$ is empty.

For the proof of Theorem 1 we rely heavily on the following lemma. The proof will be given at the end of the paper.

LEMMA 1. Let $M$ be a complete simply connected manifold with sectional curvatures $k<-1$. Let $F_{1}, \ldots, F_{n} \subset M$ be convex sets, $F=\bigcup_{i=1}^{n} F_{i}$ and denote by Chull $(F)$ the closed (in $M$ ) convex hull of $F$. Then there is a constant $C$ depending on the sets $F_{1}, \ldots, F_{n}$, such that for every $P \in \operatorname{Chull}(F)$, dist $(P, F)<C+\ln (n)$.

Of course, the nontrivial part is when the sets $F_{1}, \ldots, F_{n}$ are unbounded. This shows that the convex hull of finitely many convex sets is not much larger than the union of the sets. Namely, the convex hull cannot contain "new" ideal points, that is

$$
\operatorname{cl}\left(\operatorname{Chull}\left\{F_{1}, \ldots, F_{n}\right\}\right) \cap S_{\infty}(M)=\bigcup_{i=1}^{n}\left(\operatorname{cl}\left(F_{i}\right) \cap S_{\infty}(M)\right),
$$

where $\operatorname{cl}(K)$ denotes the closure of $K$ in $\bar{M}$.

\section{Proof of Theorem 1}

Since we are going to work on the ideal boundary it will be convenient to introduce the following notation. For a set $F \subset \bar{M}$ we denote by $F_{\infty}$ the ideal part of $F$, that is, $F_{\infty}=F \cap S_{\infty}(M)$.

In the proof of Theorem 1 we are going to use the following proposition.

Proposition 1. Let $A \subset S_{\infty}(M)$ be an open subset of $S_{\infty}(M)$ and $F \subset \bar{M}$ be a closed convex set such that $F_{\infty} \supset \partial A$, where $\partial A$ denotes the boundary of $A$ in $S_{\infty}(M)$. Then there is a closed (in $\bar{M}$ ) convex set $F^{\prime} \supset F \cup A$ such that $F_{\infty}^{\prime}=A \cup F_{\infty}$.

Proof of Proposition 1: Since we are working on the compactified manifold $\bar{M}$ we adopt the convention that geodesics will include their initial and terminal ideal points. Recall also that since the curvature is bounded away from 0 there is always a unique geodesic connecting two ideal points (see [8]). 
Define $F^{\prime} \subset \bar{M}$ to be the collection of (not necessarily finite) geodesic segments with initial and terminal points in $A \cup F$. Since $A \cup F$ is closed in $\bar{M}$ it is obvious that $F^{\prime}$ is closed as well and that $F_{\infty}^{\prime}=F_{\infty} \cup A$.

It remains to show that $F^{\prime}$ is convex. Let $P, Q \in F^{\prime}$ be two arbitrary points, ideal or otherwise. This means that there are points $a_{1}, a_{2}, b_{1}, b_{2} \in A \cup F$ such that $P$ and $Q$ lie on the geodesic segments $\left[a_{1}, a_{2}\right]$ and $\left[b_{1}, b_{2}\right]$, respectively. We want to show that the geodesic segment $[P, Q]$ lies in $F^{\prime}$. Essentially there are three cases to consider:

(a) $a_{1}, b_{1} \in A$ and $a_{2}, b_{2} \in F$,

(b) $a_{1}, b_{1}, a_{2} \in A$ and $b_{2} \in F$,

(c) $a_{1}, a_{2}, b_{1}, b_{2} \in A$.

The rest of the cases can be easily reduced to these three.

Case (a). If $P=a_{2}$ and $Q=b_{2}$ then we are done. Therefore, without loss of generality, we may assume that $P \neq a_{2}$. Consider the family of geodesic rays with initial point $a_{2}$ passing through the points of the geodesic segment $[P, Q]$. The terminal points of these rays trace out a continuous curve in $S_{\infty}(M)$ issuing from $a_{1}$. If this curve remains in the set $A$ then by definition $[P, Q] \subset F^{\prime}$ and we are done. Otherwise, denote by $P^{\prime}$ the first point on $[P, Q]$ (the point closest to $P$ ) such that the geodesic segment through $P^{\prime}$ (with initial point $a_{2}$ ) terminates in $\partial A$. By definition the geodesic segment $\left[P, P^{\prime}\right) \subset F^{\prime}$ and since $\partial A \subset F$ we see that $P^{\prime} \in F$. If $Q=b_{2}$ then we are done. Otherwise, repeat the previous procedure with $b_{2}$ and $Q$ in place of $a_{2}$ and $P$. Then we have the point $Q^{\prime} \in\left[P^{\prime}, Q\right]$ such that $Q^{\prime} \in F$ and $\left[Q^{\prime}, Q\right] \in F^{\prime}$. Since $F$ is convex we conclude that $\left[P^{\prime}, Q^{\prime}\right] \subset F$ which completes the proof of case (a).

Case (b). Without loss of generality we may assume that $P \neq a_{1}$. Similarly to the previous case we consider the family of geodesic rays with initial point $a_{1}$ passing through the points of the geodesic segment $[P, Q]$. The terminal points of these rays trace out a continuous curve on $S_{\infty}(M)$ issueing from $a_{2}$. If this curve remains in the set $A$ then by definition $[P, Q] \subset F^{\prime}$ and we are done. Otherwise, denote by $P^{\prime}$ the first point on $[P, Q]$ (the point closest to $P$ ) such that the geodesic segment through $P^{\prime}$ (with initial point $a_{1}$ ) terminates in $\partial A$ and denote by $a_{2}^{\prime}$ this terminal point. By definition the geodesic segment $\left[P, P^{\prime}\right] \subset F^{\prime}$. Since $a_{2}^{\prime} \in F$ we can apply the previous argument (case (a)) to show that $\left[P^{\prime}, Q\right] \subset F^{\prime}$ which completes the proof of case (b).

Case (c). Again, without loss of generality we may assume that $P \neq a_{1}$. By repeating the above argument, we can produce an ideal point $a_{2}^{\prime} \in F$ and a point $P^{\prime} \in[P, Q]$ such that $P^{\prime} \in\left[a_{1}, a_{2}^{\prime}\right]$ and $\left[P, P^{\prime}\right] \subset F^{\prime}$. Then, by case (b), we have $\left[P^{\prime}, Q\right] \subset F^{\prime}$ which concludes the proof of case (c) and the proposition as well.

The set $F^{\prime}$ constructed above is actually the closed convex hull of $F \cap A$.

Proof of Theorem 1: Since $S(M) \subset S_{\infty}(M)$ is disconnected there are open 
sets $A, B \subset S_{\infty}(M)$ such that $A \cap B=\emptyset, S(M) \subset A \cup B$ and $A \cap S(M), B \cap S(M) \neq \emptyset$. Let $K=\partial A$ denote the boundary of $A$. Then $K \subset S_{\infty}(M)$ is a compact subset and every point of $K$ is nonsingular. This means that for every point of $K$ there is an open neighbourhood (in $\bar{M}$ ) whose convex hull is not the whole manifold. By selecting a finite covering we have finitely many closed convex sets (in $\bar{M}$ ), denoted by $F_{1}, \ldots, F_{n} \subset \bar{M}$, such that $F_{i} \neq \bar{M}$, for $i=1, \ldots, n$ and $K \subset \bigcup_{i=1}^{n} F_{i}$. Without loss of generality we may assume that it is a minimal covering of $K$, that is, $K \not \subset \bigcup_{i \neq j} F_{i}$, for $j=1, \ldots, n$. Let $F=\operatorname{cl}\left(\operatorname{Chull}\left(\bigcup_{i=1}^{n} F_{i}\right)\right)$ denote the closed (in $\left.\bar{M}\right)$ convex hull of these sets. According to Lemma 1 we have

$$
F_{\infty}=\bigcup_{i=1}^{n}\left(F_{i}\right)_{\infty}
$$

We claim that $F=\bar{M}$. Otherwise there is an open set $G \subset S_{\infty}(M)$ such that $F \cap G=\emptyset$. By shrinking $G$ if necessary, we may assume that either $G \cap A=\emptyset$ or $G \subset A$. Let us suppose first that $G \cap A=\emptyset$. Then, by Proposition 1, we have a closed convex set $F^{\prime} \subset \bar{M}$ such that $A \cup F \subset F^{\prime}$ and $F_{\infty}^{\prime}=A \cup F_{\infty}$. This implies that $F^{\prime} \cap G=\emptyset$, that is, $F^{\prime} \neq \bar{M}$. On the other hand $F^{\prime}$ is convex and contains an open neighbourhood of any point in $A$ which clearly contradicts the assumption that $A$ contains a singular point. If $G \subset A$ then we can repeat the same argument with the exterior of $A$ instead of $A$ and arrive at the same contradiction.

The fact that $F=\bar{M}$ implies that

$$
\bigcup_{i=1}^{n}\left(F_{i}\right)_{\infty}=S_{\infty}(M), \quad n \geqslant 2 .
$$

First we prove part (a). Let $F_{a}=F_{1}$ and $F_{b}=\operatorname{cl}\left(\operatorname{Chull}\left(\bigcup_{i=2}^{n} F_{i}\right)\right)$ be closed convex subsets of $\bar{M}$. By Lemma 1 we have $\left(F_{b}\right)_{\infty}=\bigcup_{i=2}^{n}\left(F_{i}\right)_{\infty}$ and since $\left\{F_{1}, \ldots, F_{n}\right\}$ was a minimal covering of $K$, we see that $K \not \subset F_{b}$, that is, $F_{b} \neq \bar{M}$. This shows that $F_{a}$ and $F_{b}$ are proper closed convex subsets of $\bar{M}$. On the other hand (1.1) implies that $\left(F_{a}\right)_{\infty} \cup\left(F_{b}\right)_{\infty}=S_{\infty}(M)$. Since $F_{a}$ and $F_{b}$ are closed convex sets a simple argument shows that $F_{a} \cup F_{b}=\bar{M}$. The existence of nontrivial bounded harmonic functions then follows from Choi's theorem, Theorem A.

The proof of part (b) is easy. Suppose that int $(S(M)) \neq \emptyset$. Then, by (1.1) and the well known theorem of Baire it follows that for some $1 \leqslant i \leqslant n$ we have $\operatorname{int}\left(F_{i}\right) \cap S(M) \neq \emptyset$. Since $F_{i}$ contains a singular point in the interior it implies that $F_{i}=\bar{M}$ which is a contradiction. This completes the proof of part (b) and the theorem as well. 


\section{Proof of Lemma 1}

Proof of Lemma 1: By an approximation theorem of Greene and Wu [9, Proposition 2.2] we may assume that each $\partial F_{i}$ is smooth. Let $h_{i}: M \rightarrow \mathbb{R}^{+}$be the reparametrised distance function to $F_{i}$, that is, for $P \in M, h_{i}(P)=f\left(\varrho_{i}(P)\right)$, where $f(t)=1-e^{-t}$ and $\varrho_{i}(P)=\operatorname{dist}\left(P, F_{i}\right)$. Then for the differential and the Hessian of $h_{i}$ we have

$$
d h_{i}=f^{\prime} d \varrho_{i}, \quad D^{2} h_{i}=f^{\prime \prime} d \varrho_{i} \otimes d \varrho_{i}+f^{\prime} D^{2} \varrho_{i} .
$$

Denoting $h=h_{1}+\ldots+h_{n}$, we shall show that for a sufficiently small $\varepsilon>0$ the set $\widehat{F}=\{P \in M: h(P) \leqslant n-\varepsilon\}$ is convex. This implies the theorem because Chull $(F) \subset \widehat{F}$ and for every $P \in M, h(P)=n-\varepsilon$ we have the following inequality

$$
\ln \frac{1}{\varepsilon} \leqslant \operatorname{dist}(P, F) \leqslant \ln \frac{n}{\varepsilon}
$$

We prove the convexity of $\widehat{F}$ by showing that $\partial \widehat{F}$, which is the level set $h=n-\varepsilon$, has positive definite second fundamental form.

Let $\alpha>0$ be a fixed "small" angle such that

$$
\sin ^{2} \alpha<1 / 2 \text { and } \cos ^{2} \alpha>3 / 4 .
$$

Then, due to the negativity of the sectional curvatures, from a large enough distance $C_{1}$ (which depends only on the upper bound of the curvature, $k \leqslant-1$ ) the viewing angle of every convex set will be less than $\alpha / 3$, as measured by the maximal angle subtended by two points in the set. By selecting a point $P_{i} \in F_{i}$ from each convex set we can find a distance $C_{2}$ such that the viewing angle of the set $\left\{P_{1}, \ldots, P_{n}\right\}$ is smaller than $\alpha / 3$ from this distance. Combining these together we have that from the distance $\max \left\{C_{1}, C_{2}\right\}$ the viewing angle of $F$ is less than $\alpha$.

This means, in view of (2.2), that there is $\varepsilon>0$ such that for every point $P \in M$ for which $h(P)=n-\varepsilon$ we have $\measuredangle\left(\nabla h_{i}(P), \nabla h_{j}(P)\right)<\alpha$ which implies that

$$
\measuredangle\left(\nabla h_{i}(P), \nabla h(P)\right)<\alpha .
$$

Here the symbol $\measuredangle\left(\nabla h_{i}(P), \nabla h_{j}(P)\right)$ stands for the angle of the gradient vectors $\nabla h_{i}(P), \nabla h_{j}(P)$.

Let $X \in T_{P} M$ now be a unit vector tangent to the level set $h=n-\varepsilon$, that is, $d h(X)=0$. From the inequality above it follows that $X$ is almost tangent to the level sets of $\varrho_{i}$ passing through $P$, that is,

$$
d \varrho_{i}(X)<\sin \alpha .
$$

On the other hand standard arguments, involving Jacobi fields, show that the level sets of $\varrho_{i}$ have a definite convexity, that is, denoting by $A_{i}$ the second fundamental 
form of the level set $\varrho_{i}=r$ we have $A_{i}>\tanh (r) I d$, where $I d$ denotes the identity. Let $C_{3}$ be a constant, such that for $r>C_{3}$ we have $\tanh (r)>2 / 3$ and set $C=$ $\max \left\{C_{1}, C_{2}, C_{3}\right\}$. Then for a small enough $\varepsilon$, such that $\ln (1 / \varepsilon)=C$ (or equivalently if $r=\operatorname{dist}(P, F) \geqslant C$ see (2.2)), in view of (2.3) and (2.4), we have

$$
D^{2} \varrho_{i}(X, X)>\tanh (r) \cos ^{2} \alpha>1 / 2, \quad i=1,2, \ldots, n .
$$

This, together with (2.1), (2.3) and (2.4), implies that $D^{2} h_{i}(X, X)>0$ for $i=$ $1, \ldots, n$, therefore $D^{2} h(X, X)>0$, which concludes the proof of the theorem.

REMARK. If $\bigcap_{i=1}^{n} F_{i} \neq \emptyset$ then the constant $C$ in Lemma 1 will depend only on the upper bound of the curvature. From the proof it is obvious that $C$ depends only on $C_{1}, C_{2}$ and $C_{3}$. Out of these $C_{3}$ is an absolute constant and $C_{1}$ depends only on the upper bound of the sectional curvatures. In case $\bigcap_{1=1}^{n} F_{i} \neq \emptyset$ let $P \in \bigcap_{1=1}^{n} F_{i}$ be any point and set $P_{i}=P$ for $i=1, \ldots, n$. Then we can choose $C_{2}=0$.

\section{REFERENCES}

[1] A. Ancona, 'Convexity at infinity and Brownian motion on manifolds with unbounded negative curvature', Rev. Mat. Iberoamericana 10 (1994), 189-220.

[2] A. Ancona, 'Negatively curved manifolds, elliptic operators, and the Martin boundary', Ann. Math. 125 (1987), 495-536.

[3] M.T. Anderson, 'The dirichlet problem at infinity for manifolds of negative curvature', $J$. Differential Geom. 18 (1983), 701-721.

[4] M.T. Anderson and R. Schoen, 'Positive harmonic functions on complete manifolds of negative curvature', Ann. Math. 121 (1985), 429-461.

[5] A. Borbély, 'A note on the Dirichlet problem at infinity for manifolds of negative curvature', Proc. Amer. Math. Soc. 114 (1992), 865-872.

[6] A. Borbély, 'The nonsolvability of the Dirichlet Problem on negatively curved manifolds' (to appear).

[7] H.I. Choi, 'Asymptotic Dirichlet problems for harmonic functions on Riemannian manifolds', Trans. Amer. Math. Soc. 281 (1984), 691-716.

[8] P. Eberlain and B. O'Neill, 'Visibility manifolds', Pacific J. Math 46 (1973), 45-109.

[9] R.E.Greene and $\mathrm{H}$. Wu, ' $C^{\infty}$ Approximations of convex, subharmonic and plurisubharmonic functions', Ann. Sci. École Norm. Sup. 12 (1979), 69-100.

[10] Y. Kifer, 'Brownian motion and harmonic functions on manifolds of negative curvature', Theory Probab. Appl. 21 (1976), 81-95.

[11] D. Sullivan, 'The Dirichlet problem at infinity for a negatively curved manifold', J. Differential Geom. 18 (1983), 723-732.

Department of Mathematics and Computer Science

Kuwait University

Safat 13060 Kuwait

e-mail: borbely@mcc.sci.kuniv.edu.kw 